\title{
Changes in Eyelid Parameters after Orbital Decompression according to the Surgical Approach in Thyroid Eye Disease
}

\author{
Seong Ho Kim, Sung Mo Kang \\ Department of Ophthalmology and Inha Vision Science Laboratory, Inha University School of Medicine, Incheon, Korea
}

Purpose: To evaluate the changes in eyelid parameters after the endonasal approach compared with transcaruncular medial wall decompression combined with the transconjunctival inferior wall decompression approach with inferomedial strut preservation.

Methods: In total, 71 patients with thyroid eye disease who underwent orbital decompression were retrospectively evaluated. The data collected included Hertel exophthalmometry, marginal reflex distance 1, marginal reflex distance 2, interpalpebral fissure, levator function test measures, and complications related to surgery.

Results: Proptosis reduction produced a statistically significant improvement across all decompression groups. Lower eyelid retraction produced a statistically significant improvement in the two-wall decompression groups but not in the one-wall decompression groups. No statistical difference was observed in the upper eyelid retraction and levator function after surgery across all decompression groups. In the post-hoc analysis, statistical improvement was not observed in the lower eyelid retraction between the endonasal and transcaruncular approach in the one-wall and two-wall decompression groups, although statistically significant exophthalmos reduction and improvement in the lower eyelid retraction was noted in the two-wall decompression subgroups compared with one-wall decompression subgroups.

Conclusions: Our study showed no differences in eyelid parameters between the endonasal and transcaruncular decompression approaches in one-wall and two-wall decompression. In the two-wall decompression group, proptosis reduction was greater with the endonasal approach than with the transcaruncular approach when the strut was preserved; however, it does not lead to a significant difference in the improvement of lower eyelid retraction.

Key Words: Decompression, Eyelids, Graves ophthalmopathy

Graves disease is an autoimmune disease causing multisystemic involvement that includes an ophthalmologic complication called thyroid eye disease (TED). TED has

Received: March 19, 2021 Final revision: July 7, 2021

Accepted: August 4, 2021

Corresponding Author: Sung Mo Kang, MD. Department of Ophthalmology and Inha Vision Science Laboratory, Inha University School of Medicine, 27 Inhang-ro, Jung-gu, Incheon 22332, Korea. Tel: 82-32-8902400, Fax: 82-32-890-2417, E-mail: ksm0724@inha.ac.kr various manifestations, including proptosis, limited extraocular movements, eyelid changes, and visual impairment [1]. Orbital decompression is a known treatment option for these conditions [2]. Surgical techniques for orbital decompression have evolved for decades. Various approaches include bony removal of medial, inferior, or lateral wall or a combination of these techniques, which involve drastic anatomical changes in the orbit. Orbital decompression thereby affects not only changes in the degree of proptosis but also changes in the eyelid. For example, orbital decompres- 
sion has been shown to be effective in the improvement of lower eyelid retraction to some extent, but studies report inconclusive results for upper eyelid retraction [3-6]. Several recent studies have reported on whether decompression surgery with various approaches combined with eyelid surgery benefits more to the patient suffering from TED with aesthetic rehabilitation [5,7-12]. To the best of our knowledge, there is no literature comparing changes in eyelid parameters between the endoscopic and transcaruncular surgical approaches to date. In this study, we aimed to evaluate the changes in eyelid parameters after the endoscopic and transcaruncular surgical approaches to the medial wall with or without transconjunctival inferior wall decompression to evaluate whether there is any difference in the cosmetic outcomes between the two surgical approaches.

\section{Materials and Methods}

\section{Study design}

We conducted a retrospective clinical chart review of patients who underwent orbital decompression for TED between January 2015 and December 2018. All orbital decom- pression surgeries were performed by one surgeon (SMK). We included patients who underwent orbital decompression for exophthalmos caused by TED, with a clinically stable state for at least 6 months before surgery. Patients with at least 6 months of follow-up were recruited. To assess changes in eyelid parameters caused solely by decompression, patients who underwent eyelid surgery (e.g., lower eyelid retractor lysis) at the same time as orbital decompression or underwent eyelid surgery within 6 months of surgery were excluded. This retrospective study adhered to the tenets of the Declaration of Helsinki and was approved by the institutional review board of Inha University Hospital (2021-02-034). Written informed consent was waived due to the retrospective nature of this study.

To compare the endoscopic medial wall decompression approach with the transcaruncular approach, patients who underwent only medial wall decompression and those who underwent both medial wall decompression and transconjunctival inferior wall decompression were categorized into separate groups. Each group was then categorized into two subgroups according to the approach used for medial wall decompression.

Table 1. Patients' characteristics and surgical procedures

\begin{tabular}{|c|c|c|c|c|c|c|}
\hline $\begin{array}{l}\text { Baseline } \\
\text { demographics }\end{array}$ & $\begin{array}{l}\text { Endonasal } \\
\text { approach }\end{array}$ & $\begin{array}{c}\text { Transcaruncular } \\
\text { approach }\end{array}$ & $p$-value & $\begin{array}{c}\text { Endonasal + inferior } \\
\text { wall decompression } \\
\text { approach }\end{array}$ & $\begin{array}{c}\text { Transcaruncular } \\
+ \text { inferior wall } \\
\text { decompression } \\
\text { approach }\end{array}$ & $p$-value \\
\hline Patients (eyes) & $24(41)$ & $16(27)$ & NA & $17(30)$ & $14(25)$ & NA \\
\hline Age (yr) & $37.6 \pm 10.5(23-56)$ & $30.5 \pm 14.5(19-55)$ & $0.047^{*}$ & $33.4 \pm 11.9(20-60)$ & $31.6 \pm 6.9(23-54)$ & $0.472^{*}$ \\
\hline Sex & & & $0.919^{\dagger}$ & & & $0.307^{\dagger}$ \\
\hline Male & 8 & 5 & & 4 & 6 & \\
\hline Female & 33 & 22 & & 26 & 19 & \\
\hline Smoking & & & $0.798^{\dagger}$ & & & $0.519^{\dagger}$ \\
\hline Nonsmoker & $31(75.6)$ & $22(81.5)$ & & $26(86.7)$ & $19(76.0)$ & \\
\hline Exsmoker & $5(12.2)$ & $2(7.4)$ & & $2(6.7)$ & $4(16.0)$ & \\
\hline Current smoker & $5(12.2)$ & $3(11.1)$ & & $2(6.7)$ & $2(8.0)$ & \\
\hline Surgical sites & & & $0.878^{\dagger}$ & & & $0.883^{\dagger}$ \\
\hline Unilateral & $7(29)$ & $5(31)$ & & $4(24)$ & $3(21)$ & \\
\hline Bilateral & $17(71)$ & $11(69)$ & & $13(76)$ & $11(79)$ & \\
\hline Duration of GD (m) & $52.83(2-240)$ & $65.33(1-168)$ & $0.407^{*}$ & $56.60(3-121)$ & $55.36(6-140)$ & $0.895^{*}$ \\
\hline
\end{tabular}

Values are presented as number $(\%)$, mean \pm standard deviation (range), or number.

$\mathrm{NA}=$ not applicable; GD $=$ Graves disease .

${ }^{*}$ Independent $t$-test; ${ }^{\dagger}$ Chi-square test. 


\section{Patients' evaluation}

All patients in the study were assessed for demographic characteristics (Table 1) and evaluated for eyelid parameters, including Hertel exophthalmometry, margin reflex distance (MRD) 1, MRD2, interpalpebral fissure, and levator function test, with the patient's head firmly resting on the frame for the slit lamp examination in a dark room with the light source kept $3 \mathrm{~m}$ away at the same height as the patient's eye. Examiners measured the eyelid parameters using a ruler before surgery and 1,3 , and 6 months after surgery. Two examiners measured each parameter and then cross-validated the results. If the values were different, they were measured again and a consensus was reached. Digital photographs were taken before surgery and 6 months after surgery.

\section{Surgical procedures}

1) Endoscopic medial orbital wall decompression

The procedure was performed under general anesthesia. Nasal vasoconstriction was applied using oxymetazoline-saturated cottonoid pledgets and $1 \%$ lidocaine (1:100,000 epinephrine) injection to the uncinate process. Standard endoscopic intranasal uncinectomy was performed. A $45^{\circ}$ endoscopy was used in the procedure to expose the medial orbital wall as far as the orbital apex. In brief, the medial orbital wall was dissected off the periorbita and removed using a microcurette/Freer elevator as far posteriorly as the junction between the lesser wing of the sphenoid bone and the optic canal, anteriorly as the maxillary line, superiorly as the ethmoid roof, and inferiorly as the inferomedial strut between the maxillary sinus and the ethmoid sinus. The periorbita was incised from the orbital apex to the maxillary line using a sharp knife to extrude orbital fat into the ethmoid sinus.

\section{2) Transcaruncular medial wall decompression}

Patients were placed under general anesthesia, after which local anesthetic was injected in the subconjunctival plane of the lower lid and medial canthus on the affected side. An incision was made vertically via the caruncle in the medial conjunctiva, with a dissection made posteriorly via the subconjunctival tissue and then medially in the preseptal plane to the posterior lacrimal crest. The periosteum was then incised at the posterior lacrimal crest; the periorbita was reflected laterally, exposing the medial orbital wall as far posteriorly as the orbital apex, superiorly as the frontal bone, and inferiorly as the strut. The lamina papyracea was infractured, and the bone from the posterior lacrimal crest to the orbital apex and from the frontoethmoidal suture to the strut was removed. Total ethmoidectomy was then performed. The periorbita was incised anteroposteriorly along the medial orbit, and gentle globe retropulsion was performed to facilitate orbital fat herniation into the ethmoid sinus.

\section{3) Transconjunctival floor decompression}

The inferior fornix of the lower eyelid was injected with lidocaine. Next, a transconjunctival incision extending from the caruncle to the lateral canthus was made at 4-mm inferior to the inferior border of the tarsal plate. With the conjunctiva and lower eyelid retractors on superior tension, blunt dissection was performed down to the arcus marginalis at the inferior orbital rim. The periosteum was opened by monopolar cautery and elevated off the orbital floor with a malleable elevator. Bone medial to the infraorbital nerve was removed using Kerrison rongeurs, leaving the strut intact. The periorbita was incised using a sharp knife, and gentle globe retropulsion was performed to herniate inferior periorbital fat into the maxillary sinus. The conjunctiva was adequately closed with absorbable sutures.

After decompression of the medial wall, exophthalmos was measured using Hertel exophthalmometry in the operating room. An additional decompression of the inferior wall via the transconjunctival approach was performed when the exophthalmos was $>18 \mathrm{~mm}$ or the difference was $>2 \mathrm{~mm}$ from the opposite eye. The strut was preserved during medial and inferior wall decompression.

\section{Statistical analysis}

Paired sample $t$-tests were used to compare differences between preoperative and postoperative values. An independent sample $t$-test and chi-square test were used to compare demographic data. Welch's analysis of variance (ANOVA) and Games-Howell (GH) post-hoc tests were used to compare postoperative changes between the different surgical approaches because of unequal variances. Statistical analysis was conducted using PASW SPSS ver. 18.0 (SPSS Inc., Chicago, IL, USA). 


\section{Results}

This study included 71 patients and 123 eyes. The mean age at the time of orbital decompression was 35 years
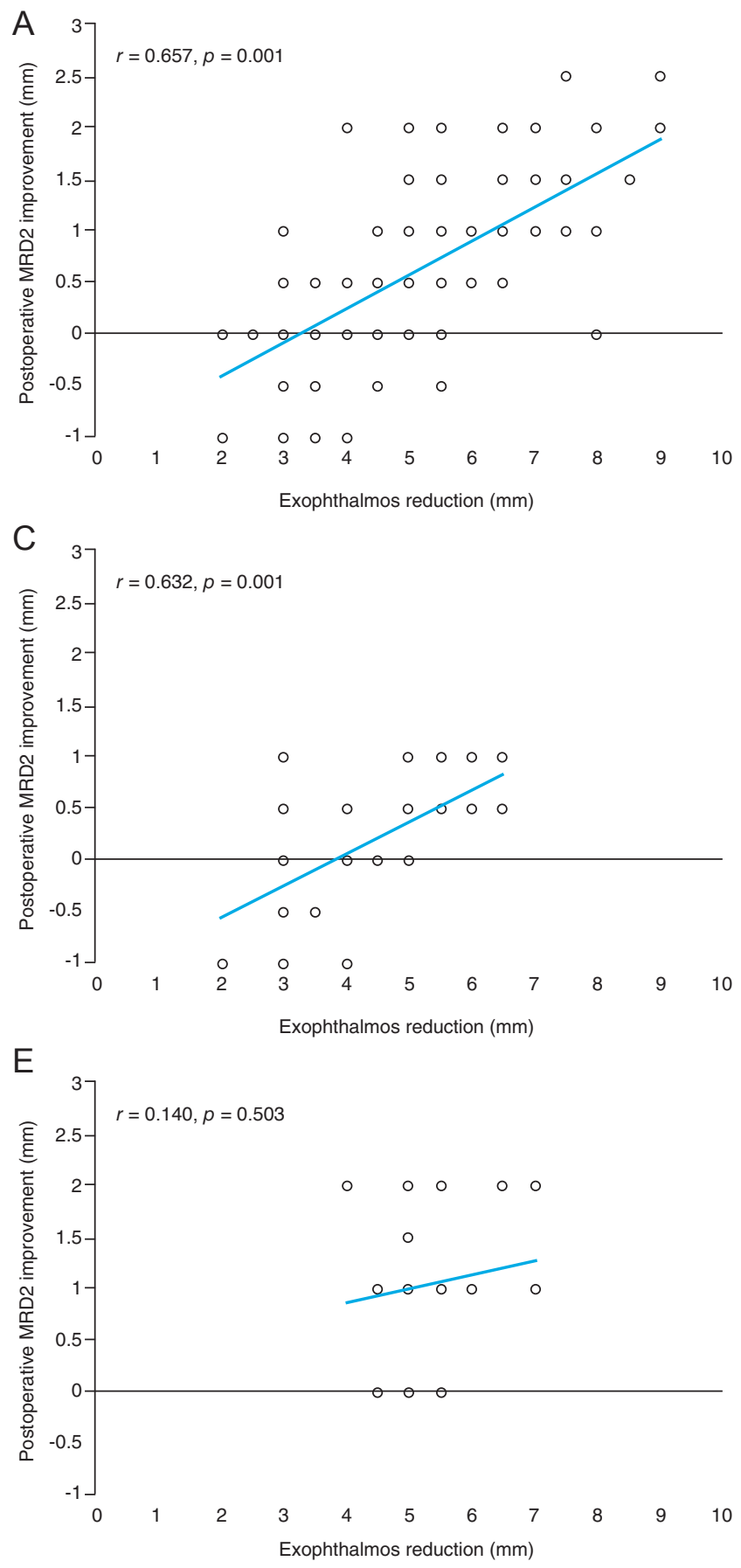

(range, 20-62 years), and 55 patients (77\%) were female. Twenty-four patients (41 eyes) underwent endonasal medial decompression, 16 patients (27 eyes) underwent transcaruncular medial decompression, 17 patients (30 eyes)
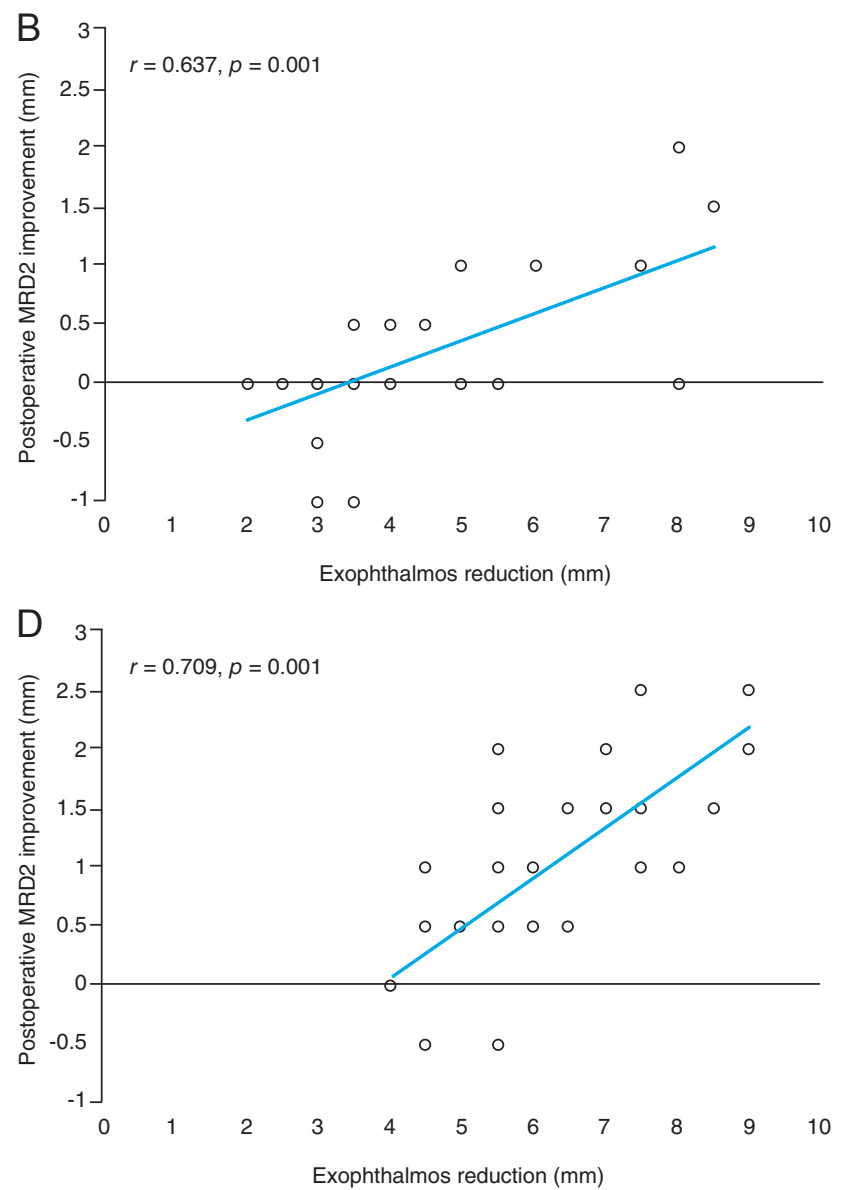

Fig. 1. Scatter plot showing linear correlation between proptosis reduction and lower eyelid elevation at 6 months postoperatively. Positive values represent postoperative improvement. (A) Entire group. (B) Endonasal approach. (C) Transcaruncular approach. (D) Endonasal + inferior wall decompression approach. $(\mathrm{E})$ Transcaruncular + inferior wall decompression approach. $\mathrm{MRD}=$ margin reflex distance. 
Table 2. Preoperative and postoperative patient data

\begin{tabular}{|c|c|c|c|c|c|}
\hline $\begin{array}{l}\text { Clinical } \\
\text { characteristics }\end{array}$ & $\begin{array}{l}\text { Endonasal } \\
\text { approach }\end{array}$ & $\begin{array}{l}\text { Transcaruncular } \\
\text { approach }\end{array}$ & $\begin{array}{c}\text { Endonasal + inferior } \\
\text { wall decompression } \\
\text { approach }\end{array}$ & $\begin{array}{l}\text { Transcaruncular } \\
\text { + inferior wall } \\
\text { decompression } \\
\text { approach }\end{array}$ & $p$-value ${ }^{*}$ \\
\hline \multicolumn{6}{|c|}{ Exophthalmometry (mm) } \\
\hline Preoperative & $21.04 \pm 1.81$ & $21.30 \pm 2.38$ & $23.78 \pm 1.99$ & $22.92 \pm 1.68$ & \\
\hline Postoperative $^{\dagger}$ & $16.88 \pm 1.59$ & $16.80 \pm 2.35$ & $17.63 \pm 1.98$ & $17.64 \pm 1.80$ & \\
\hline Difference ${ }^{\ddagger}$ & $4.16 \pm 1.69$ & $4.50 \pm 1.33$ & $6.15 \pm 1.39$ & $5.28 \pm 0.72$ & $<0.001$ \\
\hline$p$-value ${ }^{\S}$ & $<0.001$ & $<0.001$ & $<0.001$ & $<0.001$ & \\
\hline \multicolumn{6}{|l|}{ MRD1 (mm) } \\
\hline Preoperative & $3.96 \pm 1.13$ & $4.18 \pm 1.07$ & $4.60 \pm 1.00$ & $4.52 \pm 1.16$ & \\
\hline Postoperative $^{\dagger}$ & $3.86 \pm 0.97$ & $4.32 \pm 1.29$ & $4.57 \pm 0.80$ & $4.40 \pm 1.07$ & \\
\hline Difference ${ }^{\ddagger}$ & $0.10 \pm 0.80$ & $-0.13 \pm 0.63$ & $0.03 \pm 0.95$ & $0.12 \pm 0.51$ & 0.439 \\
\hline$p$-value ${ }^{\S}$ & 0.440 & 0.294 & 0.848 & 0.247 & \\
\hline \multicolumn{6}{|l|}{ MRD2 (mm) } \\
\hline Preoperative & $5.44 \pm 1.09$ & $6.41 \pm 1.58$ & $6.28 \pm 1.18$ & $7.04 \pm 1.76$ & \\
\hline Postoperative $^{\dagger}$ & $5.25 \pm 0.89$ & $6.09 \pm 1.72$ & $5.30 \pm 0.94$ & $6.00 \pm 1.92$ & \\
\hline Difference & $0.18 \pm 0.60$ & $0.22 \pm 0.64$ & $0.98 \pm 0.79$ & $1.04 \pm 0.71$ & $<0.001$ \\
\hline$p$-value ${ }^{\S}$ & 0.058 & 0.083 & $<0.001$ & $<0.001$ & \\
\hline \multicolumn{6}{|l|}{ IPF (mm) } \\
\hline Preoperative & $9.40 \pm 1.35$ & $10.59 \pm 1.60$ & $10.88 \pm 1.92$ & $11.56 \pm 1.65$ & \\
\hline Postoperative $^{\dagger}$ & $9.12 \pm 1.14$ & $10.41 \pm 2.27$ & $9.87 \pm 1.86$ & $10.40 \pm 1.89$ & \\
\hline Difference ${ }^{\ddagger}$ & $0.28 \pm 1.15$ & $0.09 \pm 0.87$ & $1.02 \pm 1.24$ & $1.16 \pm 0.89$ & $<0.001$ \\
\hline$p$-value ${ }^{\S}$ & 0.125 & 0.617 & $<0.001$ & $<0.001$ & \\
\hline \multicolumn{6}{|l|}{ LFT (mm) } \\
\hline Preoperative & $13.11 \pm 2.12$ & $13.46 \pm 2.34$ & $12.42 \pm 2.74$ & $13.94 \pm 2.32$ & \\
\hline Postoperative $^{\dagger}$ & $13.26 \pm 2.11$ & $13.64 \pm 2.38$ & $12.60 \pm 2.71$ & $14.38 \pm 2.63$ & \\
\hline Difference & $-0.15 \pm 0.44$ & $-0.18 \pm 1.37$ & $-0.18 \pm 0.84$ & $-0.44 \pm 1.65$ & 0.866 \\
\hline$p$-value ${ }^{\S}$ & 0.900 & 0.497 & 0.241 & 0.196 & \\
\hline
\end{tabular}

Values are presented as mean \pm standard deviation.

$\mathrm{MRD}=$ margin reflex distance; $\mathrm{IPF}=$ interpalpebral fissure; $\mathrm{LFT}=$ levator function test.

${ }^{*}$ Welch's analysis of variance, significant when $p<0.05$; ${ }^{\dagger}$ Postoperative parameter evaluated 6 months after surgery; ${ }^{*}$ Difference between preoperative and 6 months postoperative values; ${ }^{\S}$ Paired $t$-test $(p<0.05)$.

underwent endonasal medial and transconjunctival floor decompression, and 14 patients ( 25 eyes) underwent transcaruncular medial and transconjunctival floor decompression. Although most patients were nonsmokers, approximately $20 \%$ in each group were current or former smokers. Fifty-two patients (73\%) underwent bilateral decompression surgery, and the remaining patients underwent unilateral decompression surgery. Patients' characteristics are summarized in Table 1.

Statistically significant postoperative improvement was observed in proptosis reduction across all the decompression subgroups as well as in lower eyelid retraction and in- terpalpebral fissure between the two-wall decompression subgroups. Scatter plots correlating postoperative proptosis reduction with improvement in lower eyelid retraction are shown in Fig. 1A-1E. Significant correlation was noted between postoperative proptosis reduction and lower eyelid retraction improvement in patients who underwent orbital decompression except those in the transcaruncular + inferior wall decompression group. No significant postoperative improvement was found in MRD1 and levator function test across all decompression groups (Table 2). Based on previous studies and correlation analysis in this study indicating a correlation between MRD2 and the amount of 
Table 3. Post-hoc analysis of difference in parameters between baseline and 6 months after decompression

\begin{tabular}{lcccccc}
\hline \multirow{2}{*}{ Variable $^{*}$} & \multicolumn{4}{c}{$p$-value $^{\dagger}$} \\
\cline { 2 - 7 } & E vs. T & E vs. E + I & E vs. T + I & T vs. E + I & T vs. T + I & E + I vs. T + I \\
\hline Exophthalmometry & 0.790 & $<0.001$ & 0.002 & $<0.001$ & 0.053 & 0.023 \\
MRD2 & 0.994 & $<0.001$ & $<0.001$ & 0.001 & $<0.001$ & 0.993 \\
IPF & 0.883 & 0.063 & 0.005 & 0.013 & 0.001 & 0.959 \\
\hline
\end{tabular}

$\mathrm{E}=$ endonasal decompression approach; $\mathrm{T}=$ transcaruncular decompression approach; $\mathrm{E}+\mathrm{I}=$ endonasal + inferior wall decompression approach; $\mathrm{T}+\mathrm{I}=$ transcaruncular + inferior wall decompression approach; MRD = margin reflex distance; IPF $=$ interpalpebral fissure. ${ }^{*}$ Difference between preoperative and 6 months postoperative values; ${ }^{\dagger}$ Games-Howell test, significant when $p<0.05$.

proptosis reduction, we performed one-way ANOVA to determine changes in the eyelid parameters associated with the surgical approach. Although significant difference in proptosis reduction was found between the two-wall decompression subgroups ( $p=0.023$, Welch's ANOVA with GH test), post-hoc analysis revealed no significant difference in the lower eyelid retraction between the endonasal and transcaruncular groups in both one- and two-wall decompression subgroups ( $p=0.994$ and 0.993 , respectively; Welch's ANOVA with GH test). Two-wall decompression shows significant proptosis reduction, and post-hoc analysis between the one-wall and two-wall decompression subgroups showed significant proptosis reduction between the endonasal approach and endonasal + inferior wall, endonasal and transcaruncular + inferior wall, trancaruncular and endonasal + inferior wall approach $(p=0.001,0.002$, and 0.001, respectively; Welch's ANOVA with GH test) but not between transcaruncular and transcaruncular + inferior wall approach ( $p=0.053$, Welch's ANOVA with GH test) whereas improvement of lower eyelid retraction was found to be significant across the post-hoc analyses between the one-wall and two-wall approaches ( $p=0.383$, Welch's ANOVA with GH test) (Table 3).

No postsurgical complications were found that required eyelid surgery during the entire follow-up period. However, 1 month after surgery, one patient (6\%) exhibited a complication of diplopia in the endonasal and inferior decompression subgroups, which disappeared 3 months after surgery. All patients who underwent decompression achieved satisfactory proptosis reduction 6 months after surgery.

\section{Discussion}

Decompression via the endoscopic endonasal and transcaruncular medial wall approaches are widely accepted surgical approaches for medial wall decompression [13]. However, these approaches have several advantages and disadvantages. The endoscopic endonasal approach offers direct access to the posterior lamina papyracea and facilitates complete total ethmoidectomy [14,15], which theoretically results in a higher proptosis reduction rate than with the transcaruncular approach. However, endoscopic endonasal approach is rarely practiced by otolaryngologists, and it is difficult to implement when the patient has a nasal comorbidity, such as a deviated nasal septum. Furthermore, this approach is more frequently complicated by diplopia than other methods, particularly in decompression involving the strut $[16,17]$. By contrast, the transcaruncular approach provides easier access to the medial orbital wall. However, it may risk damage to the surrounding delicate orbital structures [18].

We focused on the change in eyelid parameters after orbital decompression because patients suffering from cosmetic rehabilitation for TED exhibited improved eyelid protrusion after orbital decompression, although cosmetic problems, such as eyelid retraction, remained [7-12]. Therefore, performing the treatment of TED in stages requires several surgeries and long-term outpatient visits to complete rehabilitation. Thus, the time and expenses to the patient and resulting stress are considerable burdens. However, the patient's burden can be greatly reduced if eyelid retraction can be simultaneously resolved by implementing an individualized orbital decompression approach tailored to the specific eyelid problem mentioned in the patient complaint.

Proposed conditions that contribute to eyelid retraction 
are generally classified as eyelid retractor hyperactivity, fibrosis, and eyeball protrusion. These three conditions interact to trigger eyelid retraction and suggest that one mechanism does not exclusively trigger eyelid retraction. In eyelid retractor hyperactivity, the upper and lower eyelid retractor becomes overexcited owing to direct stimulation of the thyroid hormone, resulting in retraction. However, this concept does not explain why eyelid retraction occurs even in euthyroid patients [19-26]. In fibrosis, the lower eyelid retractor is intrinsically connected in the capsulopalpebral fascia with the inferior rectus muscle, which is commonly affected in TED. Changes in the inferior rectus muscle dimensions or fibrosis of the capsulopalpebral fascia can cause lower eyelid retraction [22-24,27-32]. Hence, orbital decompression alone cannot completely correct eyelid retraction.

Several recent studies reported changes in eyelid parameters that occur after orbital decompression. Cumulatively, there was no improvement in upper eyelid retraction after orbital decompression and there were varied outcomes in lower eyelid retraction, depending on the number of walls on which orbital decompression was performed, with an average improvement of $0.5-1 \mathrm{~mm}$. This shows that there is some correlation between lower eyelid retraction and the degree of proptosis reduction $[5,7,8]$.

Our study showed significant correlation between postoperative proptosis reduction and lower eyelid retraction; however, there was no significant difference with respect to changes in the eyelid parameters between the endonasal and transcaruncular approaches among the one-wall and two-wall decompression subgroups. Similar to previous reports, two-wall decompression shows significant proptosis reduction and improvement of lower eyelid retraction compared with that in one-wall decompression. Interestingly, the endonasal + inferior wall approach has better proptosis reduction effect than transcaruncular + inferior wall approach. The presumed reason is that although the strut was preserved in both the endonasal and transcaruncular approaches, the effect of decompression on proptosis reduction was somewhat diminished in the transcaruncular approach and further exaggerated by inferior decompression, thereby resulting in a significant difference. However, post-hoc analysis of difference between the twowall decompression showed significant proptosis reduction but not in lower eyelid retraction. Further investigation is warranted for factors influencing the postoperative lower eyelid retraction changes apart from proptosis reduction.

The limitations of this study include the retrospective design, possible selection bias, and small sample size. In addition, when measured values of the eyelid parameters differed between the two examiners, remeasurement and discussion to reach a consensus were required. Therefore, accuracy in this study may be lower than that obtained using objective measurement methods, such as digital photo-based evaluation.

To the best of our knowledge, this is the first known study to report differences in eyelid parameters after endonasal and transcaruncular medial wall decompression combined with transconjunctival inferior wall decompression. This study did not show a correlation between the eyelid parameter and the approach of medial wall decompression. It seems that the improvement in lower eyelid retraction is mainly owing to proptosis reduction and not the surgical approach. The effect of additional inferior wall decompression on proptosis reduction was greater with the endonasal approach than with the transcaruncular approach when the strut was preserved, but it does not lead to further improvement in lower eyelid retraction. Further investigation is needed on the relationship among proptosis reduction, lower eyelid retraction, and other factors affecting postoperative lower eyelid retraction changes.

\section{Conflict of Interest}

No potential conflict of interest relevant to this article was reported.

\section{References}

1. Smith TJ, Hegedus L. Graves' disease. $N$ Engl J Med 2016;375:1552-65.

2. Leone CR Jr. The management of ophthalmic Graves' disease. Ophthalmology 1984;91:770-9.

3. Kikkawa DO, Pornpanich K, Cruz RC Jr, et al. Graded orbital decompression based on severity of proptosis. Ophthalmology 2002;109:1219-24.

4. Cho RI, Elner VM, Nelson CC, Frueh BR. The effect of orbital decompression surgery on lid retraction in thyroid eye disease. Ophthalmic Plast Reconstr Surg 2011;27:436-8.

5. Rootman DB, Golan S, Pavlovich P, Rootman J. Postopera- 
tive changes in strabismus, ductions, exophthalmometry, and eyelid retraction after orbital decompression for thyroid orbitopathy. Ophthalmic Plast Reconstr Surg 2017;33:28993.

6. Thorne AW, Rootman DB. Influence of surgical approach for decompression on lower eyelid position in thyroid eye disease. Orbit 2020;39:84-6.

7. Norris JH, Ross JJ, O’Reilly P, Malhotra R. A review of combined orbital decompression and lower eyelid recession surgery for lower eyelid retraction in thyroid orbitopathy. Br J Ophthalmol 2011;95:1664-9.

8. Fichter N, Krentz H, Guthoff RF. Functional and esthetic outcome after bony lateral wall decompression with orbital rim removal and additional fat resection in graves' orbitopathy with regard to the configuration of the lateral canthal region. Orbit 2013;32:239-46.

9. Park HH, Chun YS, Moon NJ, et al. Change in eyelid parameters after orbital decompression in thyroid-associated orbitopathy. Eye (Lond) 2018;32:1036-41.

10. Ben Simon GJ, Mansury AM, Schwarcz RM, et al. Simultaneous orbital decompression and correction of upper eyelid retraction versus staged procedures in thyroid-related orbitopathy. Ophthalmology 2005;112:923-32.

11. Bernardini FP, Skippen B, Zambelli A, et al. Simultaneous aesthetic eyelid surgery and orbital decompression for rehabilitation of thyroid eye disease: the one-stage approach. Aesthet Surg J 2018;38:1052-61.

12. Taban MR. Combined orbital decompression and lower eyelid retraction surgery. J Curr Ophthalmol 2018;30:16973.

13. Reich SS, Null RC, Timoney PJ, Sokol JA. Trends in orbital decompression techniques of surveyed american society of ophthalmic plastic and reconstructive surgery members. Ophthalmic Plast Reconstr Surg 2016;32:434-7.

14. Berhouma M, Jacquesson T, Abouaf L, et al. Endoscopic endonasal optic nerve and orbital apex decompression for nontraumatic optic neuropathy: surgical nuances and review of the literature. Neurosurg Focus 2014;37:E19.

15. Kennedy DW, Goodstein ML, Miller NR, Zinreich SJ. Endoscopic transnasal orbital decompression. Arch Otolaryngol Head Neck Surg 1990;116:275-82.

16. Michel O, Oberlander N, Neugebauer P, et al. Follow-up of transnasal orbital decompression in severe Graves' ophthalmopathy. Ophthalmology 2001;108:400-4.

17. Wright ED, Davidson J, Codere F, Desrosiers M. Endo- scopic orbital decompression with preservation of an inferomedial bony strut: minimization of postoperative diplopia. J Otolaryngol 1999;28:252-6.

18. Chang EL, Bernardino CR, Rubin PA. Transcaruncular orbital decompression for management of compressive optic neuropathy in thyroid-related orbitopathy. Plast Reconstr Surg 2003;112:739-47.

19. Waller RR. Eyelid malpositions in Graves' ophthalmopathy. Trans Am Ophthalmol Soc 1982;80:855-930.

20. Eden KC, Trotter WR. Lid-retraction in toxic diffuse goitre. Lancet 1942;240:385-7.

21. Pochin EE. The mechanism of lid retraction in Graves' disease. Clin Sci 1939;4:91-101.

22. Hawes MJ, Dortzbach RK. The microscopic anatomy of the lower eyelid retractors. Arch Ophthalmol 1982;100:1313-8.

23. Olver JM, Rose GE, Khaw PT, et al. Correction of lower eyelid retraction in thyroid eye disease: a randomised controlled trial of retractor tenotomy with adjuvant antimetabolite versus scleral graft. Br J Ophthalmol 1998;82:174-80.

24. Karesh JW, Fabrega MA, Rodrigues MM, Glaros DS. Polytetrafluoroethylene as an interpositional graft material for the correction of lower eyelid retraction. Ophthalmology 1989;96:419-23.

25. Feldman KA, Putterman AM, Farber MD. Surgical treatment of thyroid-related lower eyelid retraction: a modified approach. Ophthalmic Plast Reconstr Surg 1992;8:278-86.

26. Hedin A. Eyelid surgery in dysthyroid ophthalmopathy. Eye (Lond) 1988;2 (Pt 2):201-6.

27. Gardner TA, Kennerdell JS, Buerger GF. Treatment of dysthyroid lower lid retraction with autogenous tarsus transplants. Ophthalmic Plast Reconstr Surg 1992;8:26-31.

28. Kim JW, Ellis DS, Stewart WB. Correction of lower eyelid retraction by transconjunctival retractor excision and lateral eyelid suspension. Ophthalmic Plast Reconstr Surg 1999;15:341-8.

29. Nunery WR. Ophthalmic Graves' disease: a dual theory of pathogenesis. Ophthalmol Clin North Am 1991;4:73-87.

30. Schimek RA. Surgical management of ocular complications of Graves' disease. Arch Ophthalmol 1972;87:655-64.

31. Flanagan JC. Retraction of the eyelids secondary to thyroid ophthalmopathy: its surgical correction with sclera and the fate of the graft. Trans Am Ophthalmol Soc 1980;78:657-85.

32. Rajabi MT, Jafari H, Mazloumi M, et al. Lower lid retraction in thyroid orbitopathy: lamellar shortening or proptosis? Int Ophthalmol 2014;34:801-4. 